\title{
HRAS Mutation Analysis
}

National Cancer Institute

\section{Source}

National Cancer Institute. HRAS Mutation Analysis. NCI Thesaurus. Code C157184.

A procedure used to detect and identify mutations in the HRAS gene. 